\title{
MAGNETIC BRAKING FORMULATION FOR SUN-LIKE STARS: DEPENDENCE ON DIPOLE FIELD STRENGTH AND ROTATION RATE
}

\author{
Sean P. Matt ${ }^{1}$, Keith B. MacGregor ${ }^{2}$, Marc H. Pinsonneault ${ }^{3}$, and Thomas P. Greene ${ }^{4}$ \\ ${ }^{1}$ Laboratoire AIM Paris-Saclay, CEA/Irfu Université Paris-Diderot CNRS/INSU, F-91191 Gif-sur-Yvette, \\ France; sean.matt@cea.fr \\ ${ }^{2}$ High Altitude Observatory, National Center for Atmospheric Research (NCAR), 3080 Center Green, Boulder, CO 80301, USA; kmac@ ucar.edu \\ ${ }^{3}$ Department of Astronomy, Ohio State University, 140 West 18th Avenue, Columbus, OH 43210, USA; pinsonneault.1@ osu.edu \\ ${ }^{4}$ NASA Ames Research Center, M.S. 245-6, Moffett Field, CA 94035-1000, USA; thomas.p.greene@ nasa.gov \\ Received 2012 May 18; accepted 2012 June 5; published 2012 July 13
}

\begin{abstract}
We use two-dimensional axisymmetric magnetohydrodynamic simulations to compute steady-state solutions for solar-like stellar winds from rotating stars with dipolar magnetic fields. Our parameter study includes 50 simulations covering a wide range of relative magnetic field strengths and rotation rates, extending from the slow- and approaching the fast-magnetic-rotator regimes. Using the simulations to compute the angular momentum loss, we derive a semi-analytic formulation for the external torque on the star that fits all of the simulations to a precision of a few percent. This formula provides a simple method for computing the magnetic braking of Sun-like stars due to magnetized stellar winds, which properly includes the dependence on the strength of the magnetic field, mass loss rate, stellar radius, surface gravity, and spin rate, and which is valid for both slow and fast rotators.
\end{abstract}

Key words: magnetohydrodynamics (MHD) - stars: magnetic field - stars: rotation - stars: solar-type - stars: winds, outflows

Online-only material: color figures

\section{INTRODUCTION}

The evolution of the rotational properties of Sun-like stars exhibits numerous trends that still lack an accepted, quantitative explanation. For example, during the pre-main-sequence phase, there is not yet a comprehensive picture that explains the observed wide range of rotation rates nor the fact that a large fraction of stars rotate relatively slowly (e.g., Herbst et al. 2007; Irwin \& Bouvier 2009; Matt \& Pudritz 2005a, 2005b). During the main-sequence phase, we are still trying to understand, for example, the structure and existence of multiple "sequences" apparent in the rotation-period-versus-mass diagrams of young star clusters (e.g., Barnes 2003b; Irwin \& Bouvier 2009; Meibom et al. 2011); the apparent "saturation" of angular momentum loss in fast rotators (MacGregor \& Brenner 1991); the overall, secular spin-down of stars (Kraft 1967; Skumanich 1972; Soderblom 1983); the possibility of using rotational properties to measure stellar ages ("gyrochronology"; Barnes 2003a, 2010; Mamajek \& Hillenbrand 2008; Meibom et al. 2011; Epstein \& Pinsonneault 2012); and the correlation of stellar activity with rotation, as well as the "saturation" of this activity in fast rotators (e.g., Saar \& Brandenburg 1999; Pizzolato et al. 2003; Reiners et al. 2009; Wright et al. 2011).

We know that magnetized stellar winds are important for extracting angular momentum from stars during the main sequence (Parker 1958; Schatzman 1962; Weber \& Davis 1967; Mestel 1968) and likely during the pre-main sequence (Hartmann \& MacGregor 1982; Hartmann \& Stauffer 1989; Matt \& Pudritz 2005a). Thus, a prescription for calculating the stellar wind torque as a function of stellar parameters is a crucial ingredient in models for the rotational evolution of stars (e.g., Bouvier et al. 1997; Bouvier 2008; Denissenkov et al. 2010; Matt et al. 2012).

Reliably computing the stellar wind torque requires knowledge of the wind acceleration profile and the magnetic field geometry above the surface of the star (e.g., Mestel 1984). Until a few years ago, the only formulations available for computing stellar wind torques (e.g., Kawaler 1988) were based upon analytic or semi-analytic calculations that necessarily relied upon several simplifying assumptions, such as that of spherical symmetry and the a priori specification of the magnetic geometry, flow acceleration profile, or both. However, in real winds, all of the flow properties are determined by a complex interaction between the wind driving physics, stellar rotation, and dynamical interaction between the wind and magnetic field, and these significantly deviate from spherical symmetry. Multi-dimensional, magnetohydrodynamical (MHD) simulations provide a reliable method for computing dynamically self-consistent wind solutions, but large parameter studies are needed in order to quantify how the physics scale with various parameters. Thus, although there exists a large body of literature on the subject, reliably calculating stellar wind torques for a range of stellar and wind properties, and in a way that is useful for stellar evolution calculations, remains a challenging problem.

Using simulations of simplified, solar-like winds, Matt \& Pudritz (2008, hereafter MP08) carried out a small parameter study to determine the dependence of the wind torque on the strength of the magnetic field and mass outflow rate in the wind. ${ }^{5}$ Their formulation for the torque (Equation (15) of MP08) differed significantly from previous formulations, notably in the value of the exponent in the power-law dependence of the torque on stellar properties, such as magnetic field strength, mass loss rate, and stellar radius. This study adopted many of the same assumptions as previous analytic work-ideal MHD, a rotation-axis-aligned dipolar magnetic field, solid body stellar rotation, and spherically symmetric thermodynamic properties at the stellar surface - but the simulations did not require any assumptions about the kinematics of the flow nor how the

\footnotetext{
5 Although the results were presented in the context of pre-main-sequence angular momentum loss, the simulations of MP08 are also appropriate for main-sequence stars.
} 
magnetic geometry was modified by the flow. Thus, the MP08 torque is the most dynamically self-consistent formulation for the torque from Sun-like stars to date, and the implications for stellar evolution are still being explored.

At the same time, this formulation is derived from simulations with variations only in the magnetic field strength (relative to the mass loss rate and surface gravity). It does not fully capture the effects of different rotation rates, different thermodynamic (or energetic) properties of the wind, nor more complex magnetic geometries. Given the importance of computing stellar wind torques for a range of stellar ages (and thus a range of rotation rates), the natural next step is to extend the parameter study of MP08 to include variations in both magnetic field strength and stellar rotation rate. In this Letter, we present such a parameter study and, from these results, derive the most generally applicable stellar wind torque formula to date.

\section{SIMULATION METHOD AND PARAMETER STUDY}

We use the simulation code and method described in MP08 to compute steady-state wind solutions for Sun-like stars with a dipole magnetic field. We briefly describe the method here, and the reader will find further details in MP08 and references therein. The code solves the equations of ideal MHD under the assumption of axisymmetry and a polytropic equation of state $\left(P \propto \rho^{\gamma}\right)$. In each simulation, the numerical grid is initialized with a spherically symmetric, thermally driven Parker wind solution (Parker 1958), plus an analytic dipole magnetic field. Once the simulations begin, the wind solution relaxes to a steady state resulting from a dynamical balance between the accelerating wind and rotating magnetic field. The steady-state solution is entirely determined by the conditions that are present at the base of the wind (the "surface" of the star).

For a given initial magnetic geometry, unique wind solutions are determined by dimensionless parameters, which can be given as three velocity ratios $-v_{\mathrm{A}} / v_{\mathrm{esc}}, c_{\mathrm{s}} / v_{\mathrm{esc}}$, and $f$ specified at the surface and equator of the star-plus the adiabatic index $\gamma$. Here, $v_{\mathrm{A}}$ is the Alfvén speed, $v_{\text {esc }}$ is the gravitational escape speed, $c_{\mathrm{S}}$ is the thermal sound speed, and $f$ is the equatorial rotation speed divided by the breakup speed,

$$
\begin{gathered}
v_{\mathrm{A}} / v_{\mathrm{esc}}=B_{*}\left(4 \pi \rho_{*}\right)^{-1 / 2}\left(2 G M_{*} / R_{*}\right)^{-1 / 2}, \\
f \equiv \Omega_{*} R_{*}^{3 / 2}\left(G M_{*}\right)^{-1 / 2},
\end{gathered}
$$

where $B_{*}$ is the magnetic field strength at the stellar equator, $\rho_{*}$ is the mass density at the base of the wind, $G$ is Newton's gravitational constant, $M_{*}$ is the stellar mass, $R_{*}$ is the stellar radius, and $\Omega_{*}$ is the (solid body) angular rotation rate of the stellar surface $\left(=2 \pi / P_{*}\right.$, where $P_{*}$ is the rotation period).

The torque formulation of MP08 is based on nine simulations with variations in the parameter $v_{\mathrm{A}} / v_{\text {esc }}$. They also presented five simulations with variations in the other parameters, $f$, $c_{\mathrm{s}} / v_{\text {esc }}$, or $\gamma$, which demonstrated that these parameters affect the torque in a way that is not captured by their fit formulation. Motivated by the fact that the study of MP08 is based on a relatively small number of simulations and by the importance of precisely determining the torque for a range of stellar rotation rates, the present work extends the parameter study of MP08 to include 50 simulations sampling a large range in both $v_{\mathrm{A}} / v_{\text {esc }}$ and $f$. For all simulations presented here, we adopt a dipole magnetic geometry and fix $\gamma=1.05$ and $c_{\mathrm{s}} / v_{\mathrm{esc}}=0.222$, as in the "fiducial" case of MP08.
Table 1

\begin{tabular}{|c|c|c|c|c|}
\hline Case & $f$ & $v_{\mathrm{A}} / v_{\mathrm{esc}}$ & $\Upsilon$ & $r_{\mathrm{A}} / R_{*}$ \\
\hline 1 & 0.0000995 & 0.0753 & 27.5 & 5.15 \\
\hline 2 & 0.0000995 & 0.301 & 446 & 9.42 \\
\hline 3 & 0.0000995 & 1.51 & 13500 & 19.5 \\
\hline 4 & 0.000997 & 0.0301 & 4.11 & 3.31 \\
\hline 5 & 0.000997 & 0.0753 & 25.8 & 5.04 \\
\hline 6 & 0.000997 & 0.301 & 437 & 9.58 \\
\hline 7 & 0.000997 & 1.51 & 13000 & 19.4 \\
\hline 8 & 0.00393 & 0.0753 & 25.8 & 5.03 \\
\hline $9^{\mathrm{a}}$ & 0.00386 & 0.209 & 215 & 8.36 \\
\hline 10 & 0.00393 & 0.301 & 436 & 9.57 \\
\hline 11 & 0.00386 & 0.418 & 837 & 10.9 \\
\hline 12 & 0.00393 & 0.953 & 4900 & 15.8 \\
\hline 13 & 0.00393 & 1.51 & 13000 & 19.4 \\
\hline 14 & 0.0101 & 0.0753 & 25.6 & 5.01 \\
\hline 15 & 0.0101 & 0.301 & 432 & 9.50 \\
\hline 16 & 0.0101 & 1.51 & 12700 & 19.4 \\
\hline 17 & 0.0202 & 0.0753 & 24.9 & 4.95 \\
\hline 18 & 0.0202 & 0.301 & 417 & 9.31 \\
\hline 19 & 0.0202 & 1.51 & 11900 & 19.2 \\
\hline 20 & 0.0299 & 0.0753 & 24.0 & 4.86 \\
\hline 21 & 0.0299 & 0.301 & 395 & 9.04 \\
\hline 22 & 0.0299 & 1.51 & 10900 & 18.7 \\
\hline 23 & 0.0403 & 0.0753 & 22.8 & 4.74 \\
\hline 24 & 0.0403 & 0.301 & 367 & 8.73 \\
\hline 25 & 0.0403 & 1.51 & 9840 & 18.1 \\
\hline 26 & 0.0493 & 0.0753 & 21.6 & 4.62 \\
\hline $27^{\mathrm{a}}$ & 0.0493 & 0.235 & 212 & 7.66 \\
\hline 28 & 0.0493 & 0.301 & 341 & 8.44 \\
\hline 29 & 0.0493 & 1.51 & 8960 & 17.5 \\
\hline 30 & 0.0594 & 0.0753 & 20.1 & 4.49 \\
\hline 31 & 0.0594 & 0.301 & 312 & 8.12 \\
\hline 32 & 0.0594 & 1.51 & 8020 & 16.8 \\
\hline 33 & 0.0986 & 0.0301 & 2.70 & 2.82 \\
\hline 34 & 0.0986 & 0.0753 & 14.6 & 3.96 \\
\hline $35^{\mathrm{a}}$ & 0.0986 & 0.209 & 104 & 5.98 \\
\hline $36^{\mathrm{a}}$ & 0.0987 & 0.254 & 150 & 6.44 \\
\hline $37^{\mathrm{a}}$ & 0.0986 & 0.301 & 211 & 6.97 \\
\hline $38^{a}$ & 0.0986 & 0.363 & 295 & 7.53 \\
\hline $39^{a}$ & 0.0990 & 0.502 & 580 & 8.77 \\
\hline $40^{\mathrm{a}}$ & 0.0986 & 0.602 & 857 & 9.58 \\
\hline $41^{\mathrm{a}}$ & 0.0986 & 0.940 & 2130 & 11.8 \\
\hline $42^{\mathrm{a}}$ & 0.0986 & 2.10 & 10400 & 16.8 \\
\hline $43^{a}$ & 0.0986 & 3.01 & 20500 & 19.3 \\
\hline 44 & 0.197 & 0.0753 & 5.39 & 2.83 \\
\hline 45 & 0.197 & 0.301 & 75.9 & 4.93 \\
\hline $46^{\mathrm{a}}$ & 0.197 & 0.495 & 212 & 6.26 \\
\hline 47 & 0.197 & 1.51 & 2120 & 10.5 \\
\hline 48 & 0.403 & 0.753 & 88.0 & 4.61 \\
\hline 49 & 0.403 & 1.51 & 370 & 6.30 \\
\hline 50 & 0.403 & 3.01 & 1470 & 8.33 \\
\hline
\end{tabular}

Simulation Parameters and Results

Note. ${ }^{\text {a }}$ Same case used in the study of MP08.

Table 1 lists the input parameters $f$ and $v_{\mathrm{A}} / v_{\mathrm{esc}}$ for all 50 cases of the present study. Twelve of the simulations, as indicated in the table, are identical to those used in MP08.

\section{SIMULATION RESULTS}

From the steady-state wind simulations, we compute the total mass loss rate $\left(\dot{M}_{\mathrm{w}}\right)$ and the torque $\left(\tau_{\mathrm{w}}\right)$ directly by integrating the mass and angular momentum flux over a closed surface 


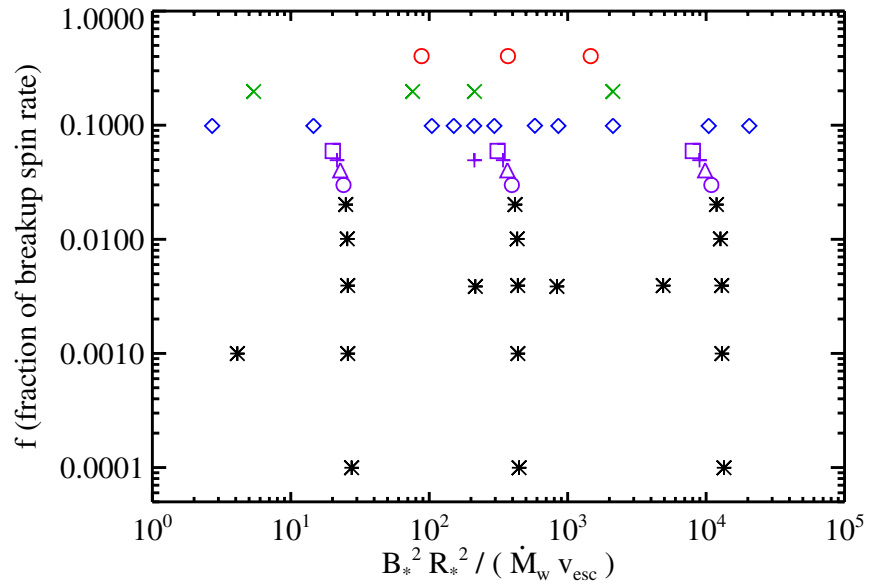

Figure 1. Parameter space explored. Each point represents a single stellar wind simulation. Symbols are as follows: asterisks represent cases with $f \lesssim 0.02$, circles are for $f \approx 0.03$ and 0.4 , triangles are for $f \approx 0.04$, pluses are for $f \approx 0.05$, squares are for $f \approx 0.06$, diamonds are for $f \approx 0.1$, and $\times$ 's are for $f \approx 0.2$.

(A color version of this figure is available in the online journal.)

containing the star. Table 1 lists the value of

$$
\Upsilon \equiv \frac{B_{*}^{2} R_{*}^{2}}{\dot{M}_{\mathrm{w}} v_{\mathrm{esc}}}
$$

for each case. The quantity $\Upsilon$ is a dimensionless way of expressing the (inverse) mass loss rate resulting from the simulations, it is physically related to the simulation parameter $\left(v_{\mathrm{A}} / v_{\mathrm{esc}}\right)^{2}$, and it is similar to the "magnetic confinement parameter" of Ud-Doula \& Owocki (2002; Ud-Doula et al. 2008, 2009). In the following analysis, we treat $\Upsilon$ as an independent variable of the simulations, even though it is a result of the simulations. We prefer to work with $\Upsilon$, as it contains the more observable/predictable quantity $\dot{M}_{\mathrm{w}}$, as opposed to $v_{\mathrm{A}} / v_{\mathrm{esc}}$, for which one must specify the density at the base of the corona $\rho_{*}$ (see Equation (1)). Furthermore, for a given value of $\rho_{*}$, the mass loss rate depends sensitively on the values of $c_{\mathrm{s}} / v_{\text {esc }}$ and $\gamma$ (see MP08), as well as the particular choice of boundary conditions, which we hold fixed in all simulations. Thus, the consideration of $\Upsilon$ as a controlling parameter (instead of $v_{\mathrm{A}} / v_{\mathrm{esc}}$ ) significantly mitigates the influence of $c_{\mathrm{s}} / v_{\mathrm{esc}}, \gamma$, and boundary condition choices in the resulting torque formulation.

Figure 1 illustrates the range of parameter space, in terms of $f$ and $\Upsilon$, spanned by all 50 simulations. Each symbol in the plot corresponds to a simulation listed in Table 1, and the different symbol styles highlight different ranges of spin rates. The asterisks are for cases in the slow rotator regime, where the rotation has a negligible influence on the wind dynamics. The remaining symbols represent cases in which rotation affects the speed and collimation, and consequently the efficiency of angular momentum loss, in the flow. For reference, the appropriate values for the solar wind are $f \approx 0.004$ and $\Upsilon$ within a possible range of $\sim 10^{2}-10^{3}$ (e.g., for $\dot{M}_{\mathrm{w}} \approx 2 \times 10^{-14} M_{\odot} \mathrm{yr}^{-1}$ and an equatorial dipole field strength of $1-5 \mathrm{G}$ ).

In order to express the resulting torques in a useful and general way, we consider the following. In a steady-state wind, under the assumptions of ideal MHD, the specific angular momentum extracted from the star is equal to $\Omega_{*} r_{\mathrm{A}}^{2}$ (e.g., Weber \& Davis 1967), where $r_{\mathrm{A}}$ is the "Alfven radius," the cylindrical radial location where the wind velocity equals the local Alfvén speed. In a three-dimensional flow, the net torque on the star can be

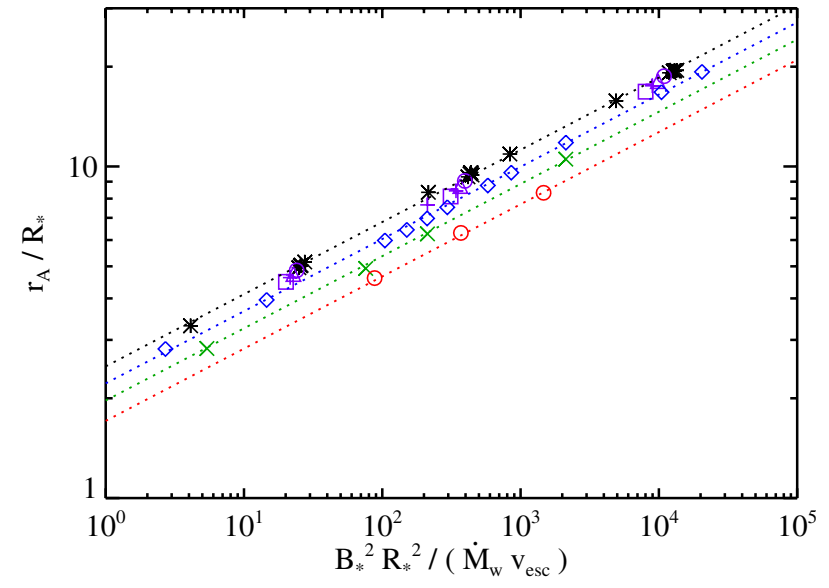

Figure 2. Alfvén radius (Equation (5)) vs. ؟. The symbols have the same meaning as in Figure 1. Dotted lines show the fit function (Equations (6) and (7)) for $f=0.001,0.1,0.2$, and 0.4 , from top to bottom, which approximately correspond to a few of the rotation rates used in the parameter study.

(A color version of this figure is available in the online journal.)

written as

$$
\tau_{\mathrm{w}}=\dot{M}_{\mathrm{w}} \Omega_{*} r_{\mathrm{A}}^{2},
$$

where $r_{\mathrm{A}}^{2}$ is an average value (Washimi \& Shibata 1993). From the simulations, we compute $\tau_{\mathrm{w}}$ and $\dot{M}_{\mathrm{w}}$ directly, so we use Equation (4) to define the dimensionless Alfvén radius as

$$
\frac{r_{\mathrm{A}}}{R_{*}} \equiv\left(\frac{\tau_{\mathrm{w}}}{\dot{M}_{\mathrm{w}} \Omega_{*} R_{*}^{2}}\right)^{1 / 2},
$$

which thus represents the square root of the dimensionless torque and is unique for a given set of dimensionless simulation parameters. Table 1 lists the resulting values of $r_{\mathrm{A}} / R_{*}$ for each case.

\section{NEW TORQUE FORMULATION}

Figure 2 shows the value of the dimensionless Alfvén radius versus $\Upsilon$ for the entire parameter study. The study of MP08 found that the dimensionless Alfvén radius was well represented by a single power law in $\Upsilon$, for all cases with the same spin rate. In other words, nine simulations in MP08 were fit by $r_{\mathrm{A}} / R_{*}=K \Upsilon^{m}$, where $K$ and $m$ were dimensionless fit parameters. In Figure 2, it is evident that the effect of rotation is to modify the value of $K$, but not to significantly affect the exponent $m$ (represented by the slope in the log-log plot). Thus, we are able to fit the 50 simulations here, having variations in both $\Upsilon$ and $f$, with only one additional free parameter. Specifically, we find that all of the data are well fit by the function

$$
\frac{r_{\mathrm{A}}}{R_{*}}=K_{1}\left[\frac{\Upsilon}{\left(K_{2}^{2}+0.5 f^{2}\right)^{1 / 2}}\right]^{m},
$$

where $K_{1}, K_{2}$, and $m$ are dimensionless fit constants. The best-fit values give

$$
K_{1}=1.30, \quad K_{2}=0.0506, \quad m=0.2177 .
$$

These values differ only by a few percent from the equivalent fit parameters of MP08 ( $m$ and $K$ ) but should be considered to 


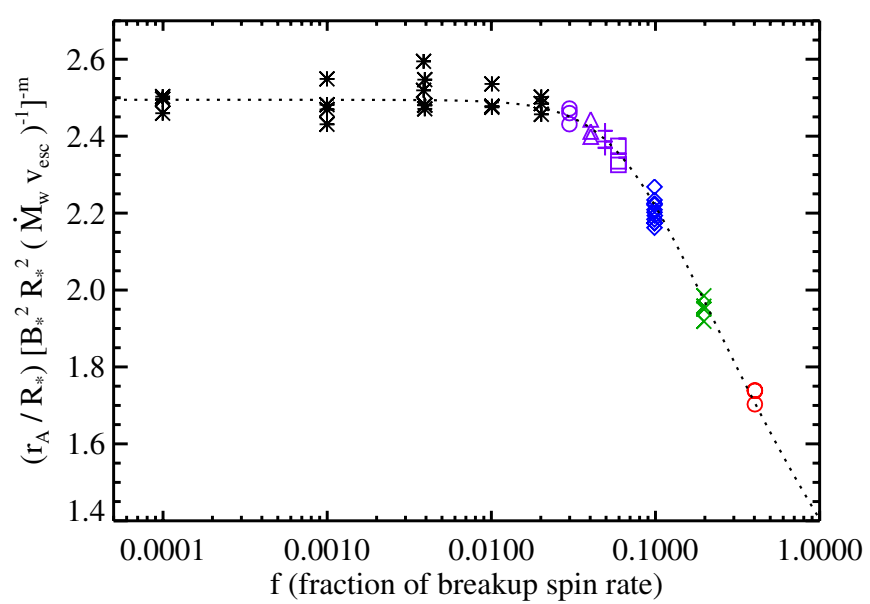

Figure 3. Dimensionless Alfvén radius times $\Upsilon^{-m}$ vs. $f$. This figure demonstrates the dependence of the Alfvén radius on the stellar spin rate, for a fixed value of $\Upsilon$. The symbols have the same meaning as Figure 1, and the dotted line shows the fit function (Equations (6) and (7)).

(A color version of this figure is available in the online journal.)

be more precise here, due to the larger number of simulations fit. Also, the functional form of Equation (6) simultaneously quantifies the effect of the wind magnetization $(\Upsilon)$ and the spin rate $(f)$ on the effective Alfvén radius. The dotted lines in Figure 2 show Equation (6) for four different values of $f=$ $0.001,0.1,0.2$, and 0.4 and using the best-fit values (7). Each dotted line goes through the symbols that represent simulations with corresponding spin rates, illustrating how well Equation (6) fits the simulation results.

One can understand the functional form of Equation (6) as follows. The most important factor for determining the Alfvén radius is the strength of the magnetic field compared to the inertia in the flow, $\Upsilon$. The MHD simulations self-consistently capture how the wind accelerates, how the magnetic field strength varies with distance from the star, and how much of the total surface magnetic flux will participate in the wind (the remaining flux exists as closed magnetic loops). The fit values of $K_{1}$ and $m$ quantify how these processes depend upon the value of $\Upsilon$, for a fixed rotation rate.

For different rotation rates, the Alfvén radius may be modified further because rotation can act as an additional wind driving component. When the stellar rotation is very slow, the rotation has a negligible effect on the wind driving. However, for fast rotation, magnetocentrifugal effects increase the wind velocity. In order to quantify this effect, one can think of the wind speed as being proportional to a rotation-modified speed,

$$
v_{\mathrm{mod}}^{2}=K_{2}^{2} v_{\mathrm{esc}}^{2}+\Omega_{*}^{2} R_{*}^{2}=v_{\mathrm{esc}}^{2}\left(K_{2}^{2}+0.5 f^{2}\right) .
$$

In this sense, Equation (6) is equivalent to the Alfvén radius being a simple power law in Equation (3), but with the factor of $v_{\text {esc }}$ being replaced by $v_{\text {mod }}$. The dimensionless factor $K_{2}$ determines at what spin rate the stellar rotation becomes dynamically important for the wind.

The effect of the stellar spin rate on the Alfvén radius (for a fixed value of $\Upsilon$ ) is best illustrated by Figure 3 . It is clear that when the spin rate is below a few percent of the breakup rate, the Alfvén radius is independent of spin rate. Faster rotation decreases the Alfvén radius. For the fastest spin rates in the parameter study ( $f \approx 0.4$ ), the Alfvén radius is decreased by a factor of approximately $30 \%$, relative to the slowly rotating

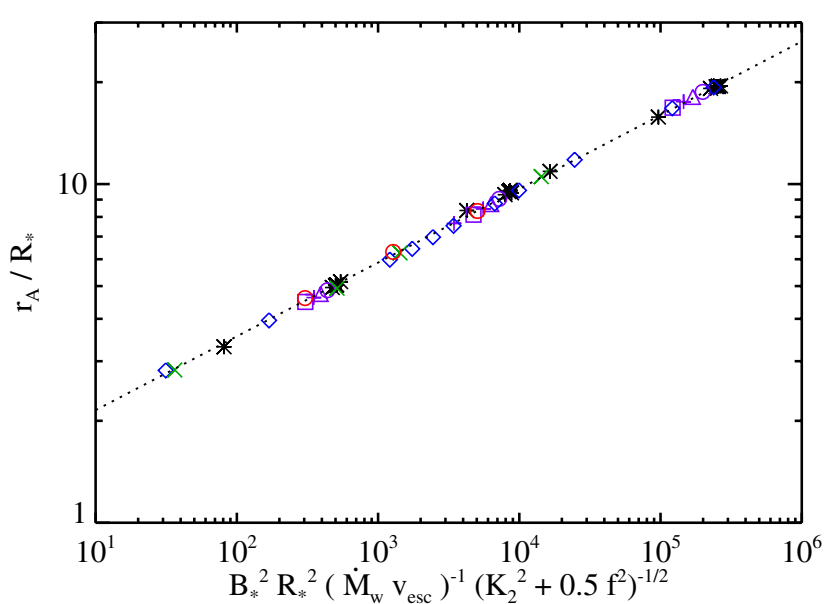

Figure 4. Dimensionless Alfvén radius vs. the quantities in square brackets in Equation (6). This figure demonstrates the dependence of the Alfvén radius on $\Upsilon$, for a fixed stellar spin rate. The symbols have the same meaning as Figure 1, and the dotted line shows the fit function (Equations (6) and (7)).

(A color version of this figure is available in the online journal.)

regime. This corresponds to a factor of approximately 2 in the torque $\left(\tau_{\mathrm{w}} \propto r_{\mathrm{A}}^{2}\right)$.

Figure 4 shows the power-law dependence of the Alfvén radius on $\Upsilon$, for a fixed value of $f$. It is remarkable how well Equation (6) fits the dimensionless Alfvén radii determined from all 50 simulations. As can be seen in Figures 2-4, all data points lie within a few percent (the biggest outlier is off by $4 \%$ ) of the fit function. This reflects the precision of the simulation method in determining the values of $r_{\mathrm{A}} / R_{*}$ and $\Upsilon$.

By combining Equations (2), (3), (5), and (6), the torque on the star, due to the stellar wind, can be written as

$$
\begin{aligned}
\tau_{\mathrm{w}} & =K_{1}^{2} B_{*}^{4 m} \dot{M}_{\mathrm{w}}^{1-2 m} R_{*}^{4 m+2} \frac{\Omega_{*}}{\left(K_{2}^{2} v_{\mathrm{esc}}^{2}+\Omega_{*}^{2} R_{*}^{2}\right)^{m}} \\
& =\frac{K_{1}^{2}}{\sqrt{2}} B_{*}^{4 m} \dot{M}_{\mathrm{w}}^{1-2 m} R_{*}^{4 m+1} v_{\mathrm{esc}}^{1-2 m} \frac{f}{\left(K_{2}^{2}+0.5 f^{2}\right)^{m}} \\
& =\frac{K_{1}^{2}}{(2 G)^{m}} B_{*}^{4 m} \dot{M}_{\mathrm{w}}^{1-2 m} \frac{R_{*}^{5 m+2}}{M_{*}^{m}} \frac{\Omega_{*}}{\left(K_{2}^{2}+0.5 f^{2}\right)^{m}},
\end{aligned}
$$

where we have listed three different equivalent forms for convenience. This formula is suitable for studies of the evolution of stellar angular momentum.

\section{DISCUSSION}

The torque formulation presented here differs significantly from analytic prescriptions that have been widely used. Of particular note, the preferred model of Kawaler (1988) results in a power-law dependence of the Alfvén radius on $\Upsilon$, with a power-law exponent of 0.5 . In the present work, we find this exponent is approximately 0.22 , which results in a significantly different dependence of the torque on all stellar parameters. A comparable exponent of between 0.2 and 0.25 was found in solar-like wind simulations of Washimi \& Shibata (1993) and Pinto et al. (2011), and Ud-Doula et al. (2009) found a similar exponent of 0.25 in simulations of radiation-driven winds from massive stars. The reasons and implications for $m \approx 0.2-0.25$, compared to the analytic work, were discussed in MP08.

We have determined the effect of the stellar rotation rate on the Alfvén radius. As evident in Figure 3, there is a slow magnetic 
rotator regime, where the rotation rate does not influence the Alfvén radius (Belcher \& MacGregor 1976). However, for faster rotation the increased wind acceleration by magnetocentrifugal effects acts to decrease the Alfvén radius. This effect and transition is not captured in previous torque formulations. Note that our simulation parameter study does not extend fully into the fast magnetic rotator regime, where the magnetocentrifugal acceleration completely dominates the thermal driving (Belcher \& MacGregor 1976). Rather, even for the fastest rotation rates considered here, the thermal wind driving was not negligible, as appropriate for solar-like winds.

The torque formulation presented here provides the most physically realistic and precise calculation to date of solar-like angular momentum loss, and it is suitable for any studies of the evolution of angular momentum of Sun-like stars. However, it has a number of limitations, which point the way for future work. First of all, the formulation is not valid in the limit of very weak magnetic fields. In particular, when the size of the Alfvén radius approaches the stellar radius, the angular momentum transport will begin to be dominated by other (e.g., viscous) effects, which are not properly included in our simulations. To take such effects approximately into account, we suggest imposing a minimum value of $r_{\mathrm{A}} \geqslant R_{*}$, in situations where weak magnetic fields may be considered.

Second, the present study fixed the physical parameters that control the thermal driving of the wind, $\gamma$ and $c_{\mathrm{s}} / v_{\mathrm{esc}}$ (which are related to the coronal temperature and heating/cooling physics). MP08 included a few simulations that had variations in these wind driving parameters, and we can also compare the torque formulation presented here with that of Ud-Doula et al. (2009), derived for radiatively driven winds. Taken together, it is clear that a reasonable range in possible wind driving physics can affect the torque on the star by a factor of $\sim 2$. It also stands to reason that the transition from slow magnetic rotator to the regime where stellar rotation is dynamically important in the wind (i.e., the value of $K_{2}$ in Equation (6)) will depend upon the wind driving physics. It will be important in future work to be able to reliably predict how the wind driving physics systematically affect the torque.

Finally, the present study assumed a rotation-axis-aligned, dipolar magnetic geometry at the stellar surface. This is justified by the fact that the largest-scale global field has the most influence on the torque, but it is not yet clear how more complex (realistic) magnetic configurations will change the scalings. MP08 presented two simulations with pure quadrupolar magnetic fields. For the same surface magnetic field strength $B_{*}$, the torque for the quadrupole case was reduced by an order of magnitude compared to the dipole case. They also noted that the power-law dependence of the Alfvén radius on the strength of the magnetic field may be altered (their two simulations suggested an exponent of 0.15 , instead of 0.22 for the dipole). Similarly, Pinto et al. (2011) simulated solar winds with a range of magnetic field complexity, noting a decreased Alfvén radius with increased magnetic complexity and suggesting a different power-law exponent when the field is significantly non-dipolar. It will be important in future work to systematically study how the torque is affected by complex, realistic magnetic geometries.

S.P.M. was supported by the NASA Postdoctoral Program at Ames Research Center, administered by Oak Ridge Associated Universities through a contract with NASA, and by the ERC through grant 207430 STARS2 (http://www.stars2.eu). The National Center for Atmospheric Research is sponsored by the National Science Foundation. T.P.G. acknowledges support from NASA's Origins of Solar Systems program via WBS 811073.02.07.01.89.

\section{REFERENCES}

Barnes, S. A. 2003a, ApJ, 586, L145

Barnes, S. A. 2003b, ApJ, 586, 464

Barnes, S. A. 2010, ApJ, 722, 222

Belcher, J. W., \& MacGregor, K. B. 1976, ApJ, 210, 498

Bouvier, J. 2008, A\&A, 489, L53

Bouvier, J., Forestini, M., \& Allain, S. 1997, A\&A, 326, 1023

Denissenkov, P. A., Pinsonneault, M., Terndrup, D. M., \& Newsham, G. 2010, ApJ, 716, 1269

Epstein, C. R., \& Pinsonneault, M. H. 2012, arXiv:1203.1618

Hartmann, L., \& MacGregor, K. B. 1982, ApJ, 259, 180

Hartmann, L., \& Stauffer, J. R. 1989, AJ, 97, 873

Herbst, W., Eislöffel, J., Mundt, R., \& Scholz, A. 2007, in Protostars and Planets V, ed. B. Reipurth, D. Jewitt, \& K. Keil (Tucson, AZ: Univ. Arizona Press), 297

Irwin, J., \& Bouvier, J. 2009, in IAU Symp. 258, The Ages of Stars, ed. E. E. Mamajek, D. R. Soderblom, \& R. F. G. Wyse (Cambridge: Cambridge Univ. Press), 363

Kawaler, S. D. 1988, ApJ, 333, 236

Kraft, R. P. 1967, ApJ, 150, 551

MacGregor, K. B., \& Brenner, M. 1991, ApJ, 376, 204

Mamajek, E. E., \& Hillenbrand, L. A. 2008, ApJ, 687, 1264

Matt, S., \& Pudritz, R. E. 2005a, ApJ, 632, L135

Matt, S., \& Pudritz, R. E. 2005b, MNRAS, 356, 167

Matt, S., \& Pudritz, R. E. 2008, ApJ, 678, 1109

Matt, S. P., Pinzón, G., Greene, T. P., \& Pudritz, R. E. 2012, ApJ, 745, 101

Meibom, S., Mathieu, R. D., Stassun, K. G., Liebesny, P., \& Saar, S. H. 2011, ApJ, 733, 115

Mestel, L. 1968, MNRAS, 138, 359

Mestel, L. 1984, Cool Stars, Stellar Systems, and the Sun, ed. S. L. Baliunas \& L. Hartmann (Lecture Notes in Physics, Vol. 193; Berlin: Springer), 49

Parker, E. N. 1958, ApJ, 128, 664

Pinto, R. F., Brun, A. S., Jouve, L., \& Grappin, R. 2011, ApJ, 737, 72

Pizzolato, N., Maggio, A., Micela, G., Sciortino, S., \& Ventura, P. 2003, A\&A, 397, 147

Reiners, A., Basri, G., \& Browning, M. 2009, ApJ, 692, 538

Saar, S. H., \& Brandenburg, A. 1999, ApJ, 524, 295

Schatzman, E. 1962, Ann. d'Astrophys., 25, 18

Skumanich, A. 1972, ApJ, 171, 565

Soderblom, D. R. 1983, APJS, 53, 1

Ud-Doula, A., \& Owocki, S. P. 2002, ApJ, 576, 413

Ud-Doula, A., Owocki, S. P., \& Townsend, R. H. D. 2008, MNRAS, 385, 97

Ud-Doula, A., Owocki, S. P., \& Townsend, R. H. D. 2009, MNRAS, 392, 1022

Washimi, H., \& Shibata, S. 1993, MNRAS, 262, 936

Weber, E. J., \& Davis, L. 1967, ApJ, 148, 217

Wright, N. J., Drake, J. J., Mamajek, E. E., \& Henry, G. W. 2011, ApJ, 743, 48 